\title{
Spin resonance strength of a localized rf magnetic field
}

\author{
S. Y. Lee \\ Department of Physics, Indiana University, Bloomington, Indiana 47405, USA
}

(Received 16 April 2006; published 20 July 2006)

\begin{abstract}
Spin-resonance strength produced by a localized rf field has been a focus of recent publications [V.S. Morozov et al., Phys. Rev. ST Accel. Beams 7, 024002 (2004).; M. A. Leonova et al. (to be published).; T. Roser, in Handbook of Accelerator Physics and Engineering, edited by A. W. Chao and M. Tigner (World Scientific, Singapore, 1999), p. 151.; M. Bai, W. W. MacKay, and T. Roser, Phys. Rev. ST Accel. Beams 8, 099001 (2005).; V. S. Morozov et al., Phys. Rev. ST Accel. Beams 8, 099002 (2005).]. This paper discusses the debated factor of 2 , and provides a formula to calculate the component enhanced by the induced betatron motion.
\end{abstract}

DOI: 10.1103/PhysRevSTAB.9.074001

\section{INTRODUCTION}

Recent spin-manipulation experiments have made efforts to understand the spin-resonance strength induced by the rf dipole or solenoidal fields in spin manipulations $[1,2]$. In particular, there is a debate on the factor of 2 in the definition of the resonance strength induced by the rf field [3-5], and the enhancement of the spin-resonance strength by more than an order of magnitude [2].

Harmonic modulation to the dipole or the solenoidal field provides a powerful tool in spin and beam manipulations [6,7]. Since the spin manipulation requires absolute knowledge of the spin-resonance strength, it is important to be able to calculate the spin-resonance strength produced by the rf fields. This report is intended to clarify the issue and derive a formula to evaluate the induced spinresonance strength. We organize the paper as follows. Section II discusses the intrinsic and the forced rf spinresonance strength. The conclusion is given in Sec. III.

\section{THE SPIN-RESONANCE STRENGTH OF A LOCALIZED RF FIELD}

In the presence of an $\mathrm{rf}$ dipole or solenoidal field modulation, the Thomas-BMT equation in the Frenet-Serret curvilinear coordinate system is

$$
\frac{d \vec{S}}{d \theta}=\vec{n} \times \vec{S}
$$

where $\vec{S}=S_{1} \hat{e}_{1}+S_{2} \hat{e}_{2}+S_{3} \hat{e}_{3}$ is the vector polarization in the orthonormal basis of the radially outward direction $\hat{e}_{1}$, the longitudinal beam direction $\hat{e}_{2}$, and the vertical direction $\hat{e}_{3}$. The dipole bending angle $\theta$ serves as the independent coordinate with $d \theta=d s / \rho$, where $s$ is the longitudinal path length along the reference orbit and $\rho$ is the radius of curvature. Here $\theta$ is constant in a straight section without dipole. The angular precession $\vec{n}$-vector is $\vec{n}=G \gamma \hat{e}_{3}-F_{1} \hat{e}_{1}-F_{2} \hat{e}_{2}$ with
PACS numbers: 29.27.Hj, 29.27.Bd, 41.75.Ak, 41.85.-p

$$
\begin{aligned}
F_{1}= & -\rho z^{\prime \prime}(1+G \gamma)+(1+G \gamma) \Theta_{1} \cos \left(\omega_{1} t+\chi_{1}\right) \\
& \times \sum_{n=-\infty}^{\infty} \delta(\theta-2 \pi n), \\
F_{2}= & (1+G \gamma) z^{\prime}-\rho(1+G)\left(\frac{z}{\rho}\right)^{\prime}+(1+G) \Theta_{2} \\
& \times \cos \left(\omega_{2} t+\chi_{2}\right) \sum_{n=-\infty}^{\infty} \delta(\theta-2 \pi n) .
\end{aligned}
$$

Here $G=g / 2-1$ is the anomalous gyromagnetic $g$-factor, $G \gamma$ is the spin precession tune, $(x, z)$ are the horizontal and vertical betatron coordinates, the prime's are derivatives with respect to the longitudinal path length $s, \Theta_{1}=B_{x} \ell / B \rho$ and $\omega_{1}$ are modulation amplitude and angular frequency of an $\mathrm{rf}$ horizontal-field dipole, and $\Theta_{2}=B_{\|} \ell / B \rho$ and $\omega_{2}$ are the modulation amplitude and angular frequency of the rf solenoidal field. The $\delta$-function indicates that the rf magnetic elements are localized kicks.

The spin-resonance strength is defined as the Fourierseries expansion:

$$
F_{1}-i F_{2}=\sum_{K} \epsilon_{K} e^{-i K \theta}
$$

A computer program DEPOL [8] can calculate the resonance strength arising from the first term of Eq. (2) and the first two terms of Eq. (3). These resonance strengths are classified into the intrinsic spin resonances, arising from the betatron motion, and the imperfection spin resonances, resulting from the beam closed orbit errors and the magnetic-field and machine-alignment errors.

Now, we consider the contribution of the spin-resonance strength produced by the $\mathrm{rf}$ modulation fields. The rf modulation fields in Eqs. (2) and (3) can perturb the spin motion in two ways. The direct effect of the rf-field on the spin motion is called the intrinsic rf spin-resonance strength. Furthermore, the rf fields can also affect orbital motion, and in turn the spin motion is affected. The result- 
ing effect is called the forced rf spin-resonance strength. We will discuss their contributions as follows.

\section{A. Intrinsic rf spin-resonance strength}

First, we consider the effect of the rf magnetic field on spin motion. Using the Fourier expansion, we find

$$
\begin{aligned}
& \cos \left(\omega_{m} t+\chi\right) \sum_{n=-\infty}^{\infty} \delta(\theta-2 \pi n) \\
& =\frac{1}{2 \pi} \sum_{n=-\infty}^{\infty} \cos \left[\left(n+\nu_{m}\right) \theta+\chi\right],
\end{aligned}
$$

where $\omega_{m}$ is the modulation angular frequency (either $\omega_{1}$ or $\left.\omega_{2}\right)$ and we have used $\omega_{m} t=\nu_{m} \theta$, where $\nu_{m}=\omega_{m} / \omega_{0}$ is the modulation tune, $\omega_{0}$ is the angular revolution frequency. Using Eq. (4), we find that the resonance strength is

$$
\left|\epsilon_{K}\right|= \begin{cases}(1+G \gamma) \frac{1}{4 \pi} \frac{B_{x} \ell}{B \rho}, & \text { dipole field modulation } \\ (1+G) \frac{1}{4 \pi} \frac{B_{\|} \ell}{B \rho}, & \text { solenoidal field modulation }\end{cases}
$$

for all sidebands at $G \gamma=n+\nu_{m}$ provided that $\nu_{m}$ is not an integer or half-integer. This is the formula provided in Refs. [3,4].

On the other hand, if the modulation tune is either a halfinteger or an integer, two revolution harmonics coherently contribute to give

$\epsilon_{K}= \begin{cases}(1+G \gamma) \frac{1}{2 \pi} \frac{B_{\chi} \ell}{B \rho} \cos \chi_{1}, & \text { dipole field modulation } \\ (1+G) \frac{1}{2 \pi} \frac{B_{\|} \ell}{B \rho} \cos \chi_{2} & \text { solenoidal field modulation, }\end{cases}$

at all sidebands. The $\cos \chi_{1}$ and $\cos \chi_{2}$ factors show that the phase of arrival time of the particle is important to kick strength. If a particle arrives at the peak of the modulation wave, i.e. $\chi_{1}$ or $\chi_{2}$ is either 0 or $\pi$, the resulting spinresonance strength is twice the strength defined by Eq. (6). If the particle arrives at the zero of the modulation wave, it will receive zero kick at all time, and the spin-resonance strength is 0 .

The factor of 2 in the resonance strength used in Eq. (4.85) of Ref. [9] reflects the fact that the modulation frequency of the solenoidal field has an integer $\nu_{m}$ for $K=$ 2 resonance and its synchrotron sidebands. The induced longitudinal electric field of the rf solenoid can act as an rf cavity to capture beam particles in the bucket with $\chi_{2}=0$ [10-12]. Spin manipulation with accelerators equipped with a snake may have a spin tune of $1 / 2$, and thus the spin modulation tune is usually also $1 / 2$. The intrinsic rf spin-resonance strength is given by the formula shown in Eq. (7).

In a storage ring with many bunches, the choice of the rffrequency for spin manipulation becomes very important. For example: a storage ring with revolution frequency $f_{0}$ and bunches filled to each bucket with harmonic number $h$, the modulation frequency must be $h f_{0} / 2$ or $h f_{0}$ in order to attain equal modulation strength for each bunch. If the spin tune is $1 / 2$, a possible modulation frequency is $h f_{0} / 2$, where the harmonic number $h$ must be an odd number. If the modulation frequency were $f_{0} / 2$, some bunches might receive zero spin kick. One can intentionally use this feature to spin flip selected bunches in a storage ring.

\section{B. Resonance strength due to the forced betatron motion}

Now, we examine the effect of the rf magnetic field on particle motion. The forced betatron or synchrotron motion can give rise to enhancement or reduction of the resonance strength. Since a pure solenoidal field produces little perturbation to betatron motion unless the horizontal and vertical betatron tunes are equal. We will not discuss its enhancement.

On the other hand, the horizontal magnetic field can produce a sizable forced betatron oscillation as shown in the Appendix [13]. The forced betatron excitation is

$$
\begin{aligned}
z_{\mathrm{ex}}(s)= & \Theta_{1} \frac{\nu_{z} \sqrt{\beta_{z}(\bar{s}) \beta_{z 0}}}{2 \pi} \sum_{n=-\infty}^{\infty} \frac{1}{\nu_{z}^{2}-\left(n+\nu_{m}\right)^{2}} \\
& \times \cos \left[\left(n+\nu_{m}\right) \phi_{z}(s)+\chi_{1}\right],
\end{aligned}
$$

where $\Theta_{1}=B_{x} \ell / B \rho$ is the amplitude of the rf-dipole kick angle, $\beta_{z 0}$ is the betatron amplitude function at the $\mathrm{rf}$ kicker location. The resonance strength at harmonic $K$ is

$$
\begin{aligned}
\epsilon_{K}= & \frac{1}{2 \pi} \int_{0}^{2 \pi}\left\{-\rho z_{\mathrm{ex}}^{\prime \prime}(1+G \gamma)\right. \\
& \left.-i\left[(1+G \gamma) z_{\mathrm{ex}}^{\prime}-\rho(1+G)\left(\frac{z_{\mathrm{ex}}}{\rho}\right)^{\prime}\right]\right\} e^{i K \theta} d \theta
\end{aligned}
$$

Such an integral can easily be evaluated in DEPOL [8]. We will derive an approximate expression for a simple accelerator lattice as follows.

The forced betatron motion is dominated by the harmonic $n$ such that $\tilde{\nu}_{m} \equiv\left|n+\nu_{m}\right| \approx \nu_{z}$. For example, for a proton beam with $G=1.7928$ and $\gamma=2.45$, we have $G \gamma=4.4$. We consider the case that $\nu_{m}=0.6$, and $\nu_{z}=$ 3.575. The most important forced betatron oscillation is located at $n=3$ with $\tilde{\nu}_{m}=3+\nu_{m}=3.6$. On the other hand, for a deuteron beam with $G=-0.14301, \gamma=$ 1.3985 , and $G \gamma=-0.20$, we can choose $\nu_{m}=0.80$. If the vertical betatron tune of the accelerator is $\nu_{z}=3.60$, both $\tilde{\nu}_{m}=3+\nu_{m}=3.80$ and $\tilde{\nu}_{m}=\left|-4+\nu_{m}\right|=3.20$ terms may contribute to the forced betatron oscillation. The first term is 0.20 unit away from $\nu_{z}$, and the second term is 0.40 unit away. The amplitudes of these two components only differ by a factor of 2 , and thus they should both be included in the calculation. When $\nu_{m}$ is an integer or a halfinteger, two harmonics may contribute equally to the forced betatron excitation 
Since Eq. (9) is linear in $z_{\mathrm{ex}}$, we can separately calculate the contribution of each term. We consider a forced betatron oscillation term:

$$
z_{\mathrm{ex}}(s)=\Theta_{1} \frac{\nu_{z} \sqrt{\beta_{z}(s) \beta_{z 0}}}{2 \pi\left(\nu_{z}^{2}-\tilde{\nu}_{m}^{2}\right)} \cos \left[\tilde{\nu}_{m} \phi_{z}(s)+\chi_{1}\right] .
$$

The integral in Eq. (9) is dominated by the first term in the bracket. Using the relation $z^{\prime \prime}+K_{z}(s) z=0$, where $K_{z}(s)$ is the focusing function, we find

$$
\begin{aligned}
\epsilon_{K}^{\mathrm{forced}} \approx & \frac{(1+G \gamma) \Theta_{1} \nu_{z} \sqrt{\beta_{z 0}}}{4 \pi^{2}\left(\nu_{z}^{2}-\tilde{\nu}_{m}^{2}\right)} \oint K_{z}(s) \\
& \times \sqrt{\beta_{z}(s)} \cos \left[\tilde{\nu}_{m} \phi_{z}(s)+\chi_{1}\right] e^{i K \theta} d s .
\end{aligned}
$$

Now, we consider an accelerator lattice made of $P$ superperiods with $M$ FODO cells in each superperiod, where each FODO cell is made of the focusing and defocusing quadrupoles separated by dipole magnets. These $M$ FODO-cell units are connected by insertion sections without dipoles. Nonzero resonance strength only occurs at $K=k P \pm \tilde{\nu}_{m}(k=$ integer $)$, given approximately by

$$
\begin{aligned}
\epsilon_{K}^{\mathrm{forced}} \approx & \frac{(1+G \gamma) \Theta_{1} \nu_{z} \sqrt{\beta_{z 0}}}{8 \pi^{2}\left[\nu_{z}^{2}-\tilde{\nu}_{m}^{2}\right]}\left(E _ { P } ^ { + } \left\{E _ { M } ^ { + } \left[g_{F} \sqrt{\beta_{z}(F)}\right.\right.\right. \\
& \left.\left.-g_{D} \sqrt{\beta_{z}(D)} e^{i\left[\left(K+\tilde{\nu}_{B}\right) /(M P)\right] \pi}\right]+X_{\mathrm{ins}}^{+}\right\} e^{i \chi_{1}} \\
& +E_{P}^{-}\left\{E _ { M } ^ { - } \left[g_{F} \sqrt{\beta_{z}(F)}\right.\right. \\
& \left.\left.\left.-g_{D} \sqrt{\beta_{z}(D)} e^{i\left[\left(K-\tilde{\nu}_{B}\right) /(M P)\right] \pi}\right]+X_{\mathrm{ins}}^{-}\right\} e^{-i \chi_{1}}\right),
\end{aligned}
$$

where $g_{F}$ and $g_{D}$ are quadrupole strengths of the FODO cell, $\beta_{z}(F)$ and $\beta_{z}(D)$ are the values of the vertical betatron function evaluated at the horizontal focusing and defocusing quadrupole locations, $X_{\text {ins }}^{ \pm}$are contributions from the insertion, $E_{P}^{ \pm}$and $E_{M}^{ \pm}$are enhancement factors due to the $P$ superperiods and the $M$ FODO cells, respectively, i.e.,

$$
\begin{gathered}
E_{P}^{ \pm}=\left(1-e^{i 2 \pi\left(K \pm \tilde{\nu}_{m}\right)}\right) /\left(1-e^{i 2 \pi\left[\left(K \pm \tilde{\nu}_{m}\right) / P\right]}\right) \\
E_{\bar{M}}^{ \pm}=\left(1-e^{i 2 \pi\left[\left(K \pm \tilde{\nu}_{B}\right) / P\right]}\right) /\left(1-e^{i 2 \pi\left[\left(K \pm \tilde{\nu}_{B}\right) /(P M)\right]}\right),
\end{gathered}
$$

$\tilde{\nu}_{B}=\tilde{\nu}_{m} \nu_{B} / \nu_{z}$, and $\nu_{B}$ is the total betatron tune in dipole cells. Strong forced $\mathrm{rf}$ spin resonances are located at $K=$ $k \pm \tilde{\nu}_{m} \approx m P M \pm \tilde{\nu}_{\mathrm{B}}$.

The intrinsic spin-resonance strength for a particle at the normalized rms emittance $\epsilon_{N}$ is

$$
\begin{aligned}
\epsilon_{K}^{\text {intrinsic }} \approx & \frac{(1+G \gamma)}{2 \pi} \sqrt{\frac{\epsilon_{N}}{\pi \beta \gamma}} \oint K_{z}(s) \sqrt{\beta_{z}(s)} \\
& \times \cos \left[\tilde{\nu}_{z} \phi_{z}(s)+\xi\right] e^{i K \theta} d s,
\end{aligned}
$$

where $\beta \gamma$ is the relativistic Lorentz factor and $\xi$ is the phase of an rms particle. Since $\tilde{\nu}_{m} \approx \nu_{z}$, the forced rf spinresonance strength can be approximated by

$$
\begin{gathered}
\left|\epsilon_{K=k P \pm \tilde{\nu}_{m}}^{\text {forced }}\right| \approx \frac{(1+G \gamma) \Theta_{1}}{2 \pi} \frac{F}{\left|\nu_{z}-\tilde{\nu}_{m}\right|}, \\
F=\frac{\sqrt{\beta_{z 0}}}{2(1+G \gamma)} \sqrt{\frac{\pi \beta \gamma}{\epsilon_{N}}}\left|\epsilon_{K=k P \pm \nu_{z}}^{\text {intrinsic }}\right| .
\end{gathered}
$$

This means that all important forced rf spin resonances are located near all strong intrinsic spin resonances of the betatron motion.

In one recent experiment [2], the rf spin-resonance strength measured for polarized proton is strongly enhanced at $G \gamma \approx 4.39=8-\tilde{\nu}_{m}$, which is near a strong intrinsic spin resonance at $8-\nu_{z}$. On the other hand, the resonance strength for polarized deuteron at $G \gamma=-0.20$ did not observe enhancement at $k \pm \tilde{\nu}_{m}=-4+3.80$ because the betatron tune of $\nu_{z}=3.60$ was far away from the modulation tune of $\tilde{\nu}_{m}=3.80$ and furthermore there was no strong intrinsic spin resonance at this location for the COSY lattice [14]. A more accurate calculation can be carried out by using the DEPOL program [8]. Although we stated earlier that the sideband $\tilde{\nu}_{m}=3.20$ may substantially contribute to the forced betatron oscillation, its effect on the spin motion may be different. For example, if the $G \gamma=-0.20$, then the spin resonance of this component of the forced betatron motion is located at $G \gamma=3-\tilde{\nu}_{m}$. For an accelerator with superperiod $P=2$, the spin resonance at $3-\tilde{\nu}_{m}$ can arise only through the gradient error, and thus its resonance strength is much reduced.

\section{CONCLUSION}

This paper points out that the intrinsic rf spin-resonance strength depends on the modulation tune. If the modulation tune is an integer or a half-integer, the maximum intrinsic rf spin-resonance strength can be twice that of the case when the modulation tune is not an integer or a halfinteger. We also derived an approximate formula to calculate the strengths of the enhanced spin resonances, and explained the enhancement of the rf spin-resonance strength for polarized protons observed at the COSY cooler synchrotron.

In general, two nearby harmonics can also contribute to enhance or reduce the rf driven betatron oscillation. Each harmonic can be calculated independently in Eq. (11). Although we can explain the enhancement of the rf spinresonance strength for polarized protons, the reduction of the rf spin-resonance strength observed for the polarized deuteron beam in Refs. [1,2] needs detailed calculation.

Besides the forced oscillations, there is an induced betatron oscillation at the betatron tune, where $A$ and $B$ coefficients of Eq. (A3) are determined by the initial condition. The resonance strength of this induced betatron oscillation can also produce enhanced resonance strength at $K=k P \pm \nu_{z}$. If the spin manipulation does not cross the betatron resonance line, we do not need to calculate this enhanced strength. However, the induced betatron oscilla- 
tion plays an important role in overcoming intrinsic spin resonances with rf dipoles [6].

\section{ACKNOWLEDGMENTS}

This work is supported in part by grants from the U.S. Department of Energy DE-FG02-92ER40747 and the National Science Foundation NSF PHY-0552389.

\section{APPENDIX: EFFECTS OF RF-DIPOLE FIELD ON BETATRON MOTION}

In the presence of a localized rf dipole, Hill's equation is

$$
\frac{d^{2} y}{d s^{2}}+K(s) y=\Theta_{1} \cos \left(\omega_{m} t+\chi\right) \sum_{n=-\infty}^{\infty} \delta(s-n C)
$$

where $\Theta_{1}=B_{z} \ell / B \rho$ and $\omega_{m}$ are, respectively, the kick angle and the angular frequency of the $\mathrm{rf}$ dipole, $C$ is the circumference, $s$ is the longitudinal path length, and $t=$ $s / \beta c$ is the time. The periodic delta function reflects the fact that the beam particles encounter the kicker field only once per revolution.

Performing the Floquet transformation to Eq. (A1) with

$$
\eta=\frac{y}{\sqrt{\beta}}, \quad \phi=\frac{1}{\nu} \int_{0}^{s} \frac{d s}{\beta},
$$

and using $\delta(s-n C)=(1 /|d s / d \phi|) \delta(\phi-2 \pi n)$, Hill's equation becomes

$$
\frac{d^{2} \eta}{d \phi^{2}}+\nu^{2} \eta=\frac{\nu \beta_{0}^{1 / 2} \Theta_{1}}{2 \pi} \sum_{n=-\infty}^{\infty} \cos \left[\left(n+\nu_{m}\right) \phi+\chi\right]
$$

where $\nu_{m}=\omega_{m} / \omega_{0}$ is the modulation tune, $\beta_{0}$ is the value of the betatron amplitude function at the rf-dipole location, and $\omega_{0}$ is the orbital angular frequency. The solution of the inhomogeneous Hill's equation is

$$
\eta=\eta_{\mathrm{co}}+A \cos \nu \phi+B \sin \nu \phi+\eta_{\mathrm{ex}},
$$

where $A$ and $B$ are the amplitudes of the induced betatron motion determined by the initial condition, and the particular solution $\eta_{\mathrm{ex}}$ is the time dependent forced oscillation term:

$$
\eta_{\mathrm{ex}}=\sum_{n=-\infty}^{\infty} \frac{\nu \beta_{0}^{1 / 2} \Theta_{1}}{2 \pi\left[\nu^{2}-\left(n+\nu_{m}\right)^{2}\right]} \cos \left[\left(n+\nu_{m}\right) \phi+\chi\right] .
$$

Note that the discrete nature of the localized kicker generates error harmonics $n+\nu_{m}$ for all $n \in(-\infty, \infty)$.
For example, if the modulation tune is $\nu_{m}=0.8$, the $\mathrm{rf}$ field produces sidebands $\left|n+\nu_{m}\right|=0.2,0.8,1.2,1.8, \ldots$ around the revolution lines. The most important forced betatron oscillation is located at $\tilde{\nu}_{m} \equiv\left|n+\nu_{m}\right| \approx \nu$. In some cases, two nearby harmonics can simultaneously contribute to the forced betatron oscillation.

[1] V.S. Morozov et al., Phys. Rev. ST Accel. Beams 7, 024002 (2004).

[2] M. A. Leonova et al. (to be published).

[3] T. Roser, in Handbook of Accelerator Physics and Engineering, edited by A.W. Chao and M. Tigner (World Scientific, Singapore, 1999), p. 151.

[4] M. Bai, W. W. MacKay, and T. Roser, Phys. Rev. ST Accel. Beams 8, 099001 (2005).

[5] V.S. Morozov et al., Phys. Rev. ST Accel. Beams 8, 099002 (2005).

[6] M. Bai et al., Phys. Rev. E 56, 6002 (1997); M. Bai et al., Phys. Rev. Lett. 80, 4673 (1998).

[7] M. Syphers et al., Phys. Rev. Lett. 71, 719 (1993); D. Li et al., Phys. Rev. E 48, R1638 (1993); H. Huang et al., Phys. Rev. E 48, 4678 (1993); Y. Wang et al., Phys. Rev. E 49, 1610 (1994); Y. Wang et al., Phys. Rev. E49, 5697 (1994); S. Y. Lee, Phys. Rev. E49, 5706 (1994); S. Y. Lee et al., Phys. Rev. E49, 5717 (1994); D. Jeon et al., Phys. Rev. Lett. 80, 2314 (1998); M. H. Wang, and S. Y. Lee, J. Appl. Phys. 92, 555 (2002); W. Guo and S. Y. Lee, Phys. Rev. E 65, 066505 (2002).

[8] E. Courant and R. Ruth, BNL Report No. BNL-51270, 1980.

[9] S. Y. Lee, Spin Dynamics and Snakes in Synchrotrons (World Scientific, Singapore, 1997), p. 79, Eq. (4.85).

[10] V. A. Anferov et al., Phys. Rev. A 46, R7383 (1992).

[11] Bradley van Guilder, Ph.D. thesis, University of Michigan, 1993.

[12] See Refs. [10,11] for the description of the rf solenoid. The solenoid, constructed for spin-manipulation experiments at the IUCF Cooler Ring, was made of a 21-turn cooper coil wound around a ceramic vacuum chamber. The induced longitudinal electric field $\Delta V=L(d I / d t)$ within the length of the coil was limited to about $25 \mathrm{kV}$ peak to peak, where $L$ is the inductance and $d I / d t$ is the rate change of current. The longitudinal electric field is normally not important except near the $K=2$ spinmanipulation experiment. The radial polarization is supposed to be averaged to zero. A nonzero "radial" polarization is possible if the induced electric potential of the solenoid captures protons in its bucket.

[13] S. Y. Lee, Accelerator Physics (World Scientific, Singapore, 2005), 2nd ed., p. 96, with misprint corrected.

[14] A. Lehrach et al., Nucl. Instrum. Methods Phys. Res., Sect. A 439, 26 (2000). 